It is good to see these two great figures, Henderson and his successor Angus MacNiven, bracketed together. The authors could not resist reference to MacNiven's alleged eccentricity in "the popular Gartnavel mythology", and quote one of the famous tales; but some who knew MacNiven came to believe these were secretly fostered, if not indeed invented, by that eminently sane but very subtle Highland gentleman. He combined a selfless and effective devotion to his patients with a wryly witty selfdepreciation, a trait not common in distinguished doctors.
This is a good book, well written, illustrated, and extremely cheap. The history of a fine hospital may even be of some current relevance in these days of bed closures and community care. Andrews, in the very last sentence of the book, wisely writes "The benefits and drawbacks of such changes at Gartnavel Royal and elsewhere - and patients' own views of them - will, more than likely, only become clear in subsequent generations".

A. C. TAIT

Formerly Physician Superintendent

Crichton Royal Hospital, Dumfries, Scotland

\title{
List of assessors
}

The editors of the Psychiatric Bulletin would like to thank the following who have acted as specialist assessors during the past six months.

$\begin{array}{llll}\text { D. Abrahamson } & \text { J. A. T. Dyer } & \text { N. L. Holden } & \text { G. Pullen } \\ \text { C. E. Adams } & \text { K. Ebmeir } & \text { L. Ions } & \text { J. Race } \\ \text { G. Andrews } & \text { L. H. Fagin } & \text { R. G. Jezzard } & \text { F. Reader } \\ \text { L. Appleby } & \text { A. Farmer } & \text { D. Johnson } & \text { S. Ritter } \\ \text { B. Ballinger } & \text { R. Farmer } & \text { D. J. Jolley } & \text { J. Roberts } \\ \text { G. E. Berrios } & \text { P. Fenwick } & \text { O. Junaid } & \text { T. D. Rogers } \\ \text { S. Bhate } & \text { E. Finch } & \text { C. A. Kaplan } & \text { H. R. Rollin } \\ \text { D. Bhugra } & \text { J. Fisk } & \text { C. L. E. Katona } & \text { O. Russell } \\ \text { D. Black } & \text { D. G. Fowlie } & \text { R. F. Kehoe } & \text { L. Scarth } \\ \text { N. Bouras } & \text { W. Fraser } & \text { A. M. P. Kellam } & \text { D. Scott } \\ \text { P. M. A. Bowden } & \text { P. Freeling } & \text { P. Kennedy } & \text { J. L. Scott } \\ \text { W. D. Boyd } & \text { C. P. L. Freeman } & \text { G. E. Langley } & \text { M. A. Serfaty } \\ \text { W. F. Bynum } & \text { E. Garralda } & \text { R. Levy } & \text { J. Smith } \\ \text { H. R. Cattell } & \text { I. Glass } & \text { M. H. Lim } & \text { D. Somekh } \\ \text { J. Chick } & \text { A. Gath } & \text { C. S. Littlejohns } & \text { R. S. Stern } \\ \text { D. Chiswick } & \text { A. Ghodse } & \text { R. Littlewood } & \text { M. Tannahill } \\ \text { J. Coid } & \text { A. T. Grounds } & \text { R. M. Lyall } & \text { D. Tantam } \\ \text { J. L. Cox } & \text { M. Greenberg } & \text { A. A. McKechnie } & \text { D. Thompson } \\ \text { J. Cookson } & \text { J. Greenwood } & \text { F. Margison } & \text { G. J. Thornicroft } \\ \text { J. L. Crammer } & \text { A. Hall } & \text { S. Montgomery } & \text { P. Tyrer } \\ \text { A. David } & \text { M. A. Harper } & \text { J. O'Hara } & \text { J. P. Wattis } \\ \text { K. Davison } & \text { J. Higgins } & \text { R. L. Palmer } & \text { J. A. Whitehead } \\ \text { K. A. Day } & \text { K. Hawton } & \text { C. M. Parkes } & \text { S. Whyte } \\ \text { C. Dean } & \text { J. L. Herzberg } & \text { S. Payne } & \text { S. R. Wilson } \\ \text { J. Devoy } & \text { P. Hoare } & \text { A. J. Pelosi } & \text { R. Wrate } \\ \text { L. Drummond } & \text { S. R. Hirsch } & \text { J. S. Pippard } & \text { A. J. Yellowlees } \\ & & & \end{array}$

